\title{
The formation of hard TeV $\gamma$-ray spectra in blazars
}

\author{
Eva Lefa \\ Max-Planck-Institut für Kernphysik, P.O. Box 103980, 69029 Heidelberg, Germany \\ Landessternwarte, Königstuhl 12, 69117 Heidelberg, Germany \\ E-mail: eva.lefaempi-hd.mpg.de
}

\section{Frank Rieger}

Max-Planck-Institut für Kernphysik, P.O. Box 103980, 69029 Heidelberg, Germany

\section{Felix Aharonian}

Max-Planck-Institut für Kernphysik, P.O. Box 103980, 69029 Heidelberg, Germany

Dublin Institute for Advanced Studies, 31 Fitzwilliam Place, Dublin 2, Ireland

The TeV gamma-ray spectra of some BL Lacs, after being corrected for intergalactic absorption in the extragalactic background light (EBL), appears unusually hard. This finding poses challenges to conventional acceleration and emission models. We analyze the constraints to produce such hard $\mathrm{TeV}$ spectra within a time-dependent, leptonic synchrotron-Compton approach, utilizing a narrow, very energetic electron distribution.

25th Texas Symposium on Relativistic Astrophysics - TEXAS 2010

December 06-10, 2010

Heidelberg, Germany 


\section{Introduction}

Leptonic models of radiation have been popular in explaining the non-thermal continuum emission in blazars. The X-ray emission is commonly attributed to synchrotron radiation of relativistic electrons that are efficiently accelerated within the source. The Compton up-scattering of these synchrotron photons by the same electron population is thought to produce the high-energy $\gamma$-ray emission in so-called synchrotron self-Compton (SSC) models, e.g. [四, whereas in external Compton scenarios (EC) the origin of the dominant soft photon field is considered to be external to the source, see e.g. [ [ $]-$ 团].

The recent detection of VHE $\gamma$-rays from blazars with redshift $z \geq 0.1$, however, poses some challenges to the conventional SSC and EC interpretations. VHE gamma-rays emitted by such distant objects arrive after significant absorption caused by their interactions with the extragalactic background light (EBL) via the process $\gamma \gamma \rightarrow e^{+} e^{-}$[[]]. Reconstruction of the absorption-corrected intrinsic VHE $\gamma$-ray source spectra based on state-of-the-art EBL models then yields unusually hard VHE source spectra, that are difficult to account for with standard leptonic models. Two outstanding cases concern the blazars 1ES 1101-232 at $z=0.186$ [G, 团] and 1ES 0229+200 [ at $z=0.14$, where intrinsic photon indices $\Gamma \lesssim 1.5$, $(\Gamma=\alpha+1$, where $\alpha$ is the spectral index, $F_{v} \propto v^{-\alpha}$ ) have been inferred, with realistic values likely to be even lower than $\Gamma \sim 1$.

Any hard injection spectrum of electrons, even a monochromatic one, is normally expected to quickly undergo radiative (synchrotron or Thomson) cooling and thereby develop a standard $E_{e}^{-2}-$ form with corresponding IC (Thomson) $\gamma$-ray photon spectrum $E_{\gamma}^{-1.5}$. In addition, suppression of the cross-section due to Klein-Nishina effects usually leads to even steeper spectra $(\Gamma>1.5)$ at $\mathrm{TeV}$ energies.

Though there is a non-negligible uncertainty in the absolute EBL level, the intrinsic spectra are unusually hard even when one considers the lowest levels of the EBL [ [] . On the other hand and apart from the challenges arising for SSC interpretations, these VHE spectra obviously carry important information about the level of the EBL, and thus a deeper understanding of the mechanisms acting within the source seems now even more desirable.

A number of alternative explanations have been explored in the literature to overcome the problem [Q-[2]. Within the SSC context, for example, Katarzyński et al. [지] have suggested that by assuming a very large value for the minimum cut-off of the injected energetic electron distribution, hard VHE spectra $F_{V} \propto v^{1 / 3}$ can be produced.

Here we explore (see [14] for more details) the possible formation of hard VHE slopes within a time-dependent synchrotron-Compton approach. We examine two different electron distributions, a power-law with a high value for the minimum cut-off and a quasi-Maxwellian distribution.

\section{Quasi-monoenergetic electron distributions}

Within a stationary SSC approach, the hardest possible (extended) VHE spectrum is approximately $F_{v} \propto v^{1 / 3}$. This is related to the single electron synchrotron emissivity function $G(x)$, that exhibits a dependence $G(x) \propto v^{1 / 3}$ below the critical frequency $v_{c} \equiv 3 \gamma^{2} e B \sin \alpha /\left(4 \pi m_{e} c\right)$. A power-law electron distribution of index $p$ will thus emit a synchrotron spectrum with a main branch of power-law shape and index $\alpha=(p-1) / 2$, but below the minimum synchrotron fre- 
quency $\varepsilon_{\min }^{s} \propto \delta\left(B \gamma_{\min }^{2}\right)$ (with $\delta$ the Doppler factor) the radiation will be dominated by the $\gamma_{\min }-$ electrons and therefore exhibit a $F_{v} \propto v^{1 / 3}$ slope. This hard 1/3-slope may then also appear in the VHE range because the Compton spectrum mimics the behavior of the synchrotron spectrum at the lower energy part, namely below the "minimum Compton energy" $\varepsilon_{\min }^{c} \simeq \delta \gamma_{\min }^{2}\left(b \gamma_{\min }^{2}\right)$, where $b \equiv\left(B / B_{\mathrm{cr}}\right) m_{e} c^{2}, B_{\mathrm{cr}}=m_{e}^{2} c^{3} /(e \hbar)$. In the extreme Klein-Nishina $(\mathrm{KN})$ case, i.e., when up-scattering of the minimum synchrotron photons by the minimum electrons occurs in the $\mathrm{KN}$ regime so that $\frac{4}{3} b \gamma_{\min }^{3}>1$, the corresponding energy below which one can see the hard $1 / 3$-slope is $\gamma_{\min } m_{e} c^{2}$, and it approximately corresponds to the peak of the emitted luminosity (for any powerlaw electron index) which then appears particularly sharp, see. e.g., [[15]].

Inevitably, in order to get the hard $(\alpha=1 / 3)$ - slope in the TeV range, one needs high values for the low-energy cut-off of the electron distribution [[13]. This assumption has been used in Tavecchio et al. [15] to reproduce the SED of the blazar 1ES 0229+200 within a stationary SSC approach. The generic difficulty for such an approach however is (as noted above), that an energetic electron distribution is expected to quickly develop a $\gamma^{-2}$-tail below $\gamma_{\min }$ due to synchrotron cooling, thereby moving the Compton VHE slope to softer values. To overcome this problem, unusually low values for the magnetic field, $B \sim\left(10^{-4}-10^{-3}\right) \mathrm{G}$, have been suggested [प5]]. For such low values the electron distribution would remain essentially unchanged on timescales of up to a few years. Obviously, one would then not expect to observe significant variability on shorter timescales, and this may allow an important test of the model. Arguments based on magnetic flux conservation naively suggest that the magnetic field value, when scaled from the black hole region to the emission site, should be at least one or two orders of magnitude higher, so that one would need to destroy magnetic flux for such a scenario to work. On the other hand, an energetic electron distribution needed in combination with very low magnetic fields would imply a strong deviation from equipartition. The high electron pressure may in fact facilitate an expansion of the source (introducing adiabatic losses).

Thus, as far as a narrow energetic particle distribution is concerned, a relativistic Maxwellian may come as a more natural representation. Such an electron distribution can be the outcome of a stochastic acceleration process (e.g., 2nd order Fermi) that is balanced by synchrotron (or/and Compton) energy losses, or in general any energy loss mechanism that exhibits a quadratic dependence on the particle energy, see e.g., [16-[18]. The steady-state solution of the corresponding Fokker-Planck diffusion equation reads

$$
f(\gamma)=A \gamma^{2} e^{-\left(\frac{\gamma}{\gamma_{c}}\right)^{1+\alpha_{p}}}
$$

with constant $A$ to be defined by the initial conditions and $\alpha_{p}$ related to the turbulence spectrum considered [14]. The critical Lorentz factor $\gamma_{c}$ approximately corresponds to the energy at which acceleration is balanced by (synchrotron) cooling. Depending on the choice of parameters, cut-off energies $\gamma_{c} \sim 10^{5}$ may well be achieved.

The synchrotron spectrum that arises from such a Maxwellian distribution is dominated by the emission of electrons with $\gamma_{c}$ (Fig. 四). It exhibits the characteristic $1 / 3$-slope up to the corresponding "synchrotron cut-off frequency" $h v_{c}^{\text {syn }} \sim \delta b \gamma_{c}^{2}$. Thus the Compton spectrum is very similar to the one resulting from a narrow power-law if one chooses a value for the cut-off energy close to the minimum electron energy of the power-law distribution. The peak of the Compton flux then offers 
an estimation of the cut-off energy as $v_{\text {peak }}^{c} \propto \gamma_{c}$. As this is a steady-state solution including radiative losses, there is no further need to invoke extreme values for the magnetic field or the particle number density.
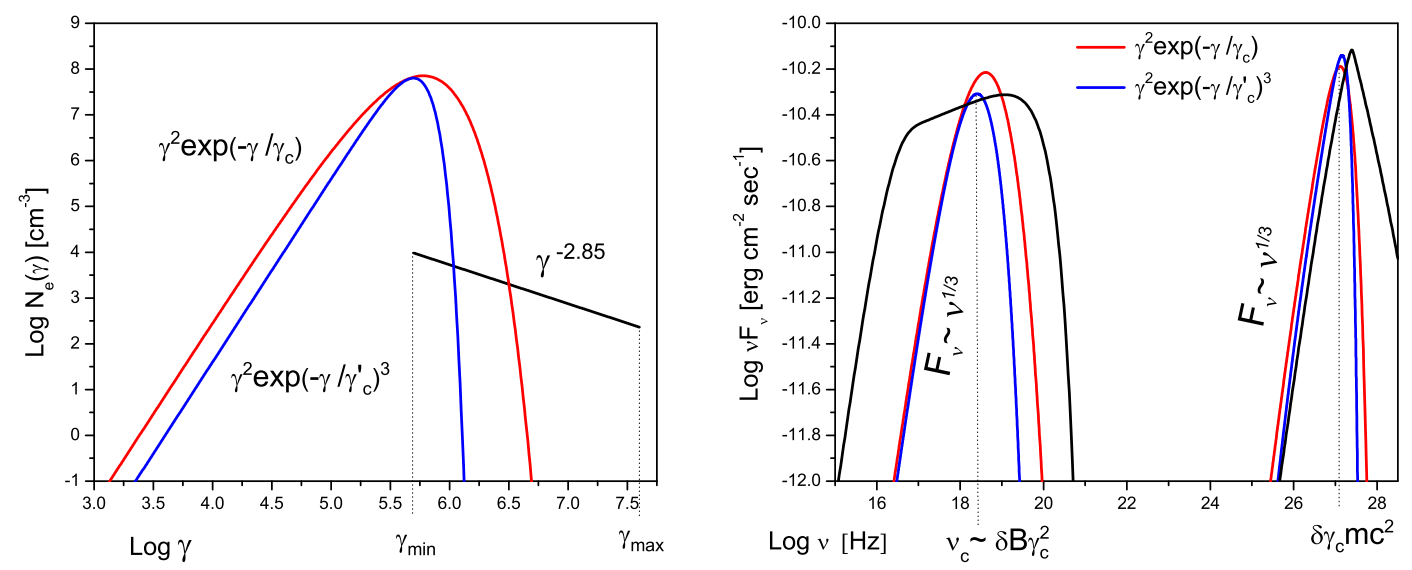

Figure 1: SSC spectrum (right panel) for different electron distributions (left panel). Black line: Power-law with large value of the minimum energy (as in Tavecchio et al. 2009). The parameters used are $\gamma_{\min }=5 \times 10^{5}$, $\gamma_{\max }=4 \times 10^{7}$, power law index $p=2.85, B=4 \times 10^{-4} \mathrm{G}, k_{e}=6.7 \times 10^{8} \mathrm{~cm}^{-3}, R=5.4 \times 10^{16} \mathrm{~cm}$ and Doppler factor $\delta=50$. Red line: Relativistic Maxwellian distribution $N_{e}=K_{e} \gamma^{2} \exp \left(-\frac{\gamma}{\gamma_{c}}\right)$ with parameters $\gamma_{c}=1.5 \times 10^{5}, B=0.07 \mathrm{G}, K_{e}=3 \times 10^{-14} \mathrm{~cm}^{-3}, R=2 \times 10^{14} \mathrm{~cm}$ and $\delta=33$. The peak of Compton flux occurs in the $\mathrm{KN}$ regime as $\left(B / B_{c r}\right) \gamma_{c}^{3} \simeq 160>>1$. Blue line: Relativistic Maxwellian distribution $N_{e}=K_{e} \gamma^{2} \exp \left(-\frac{\gamma}{\gamma_{c}}\right)^{3}$ with parameters $\gamma_{c}=5.3 \times 10^{5}, B=0.06 \mathrm{G}, K_{e}=4 \times 10^{-15} \mathrm{~cm}^{-3}, R=2 \times 10^{14} \mathrm{~cm}$ and $\delta=33$.

\section{Expansion of the source and adiabatic losses}

In the case of a power-law distribution, the expansion of the source could change the conclusions drawn above. In particular, if one assumes a very low magnetic field such that synchrotron losses are negligible, then adiabatic losses may become important and alter the electron distribution. A simple comparison of the energy loss rate for these two mechanisms reveals that adiabatic losses dominate when

$$
B(t)^{2} R(t)<\frac{6 \pi m_{e} c^{2}}{\sigma_{T}}\left(\frac{u}{c}\right) \frac{1}{\gamma}=2.3 \times 10^{19}\left(\frac{u}{c}\right) \frac{1}{\gamma},
$$

where $R(t)=R_{0}+u\left(t-t_{0}\right)$ is the radius of the source and $u$ the constant velocity of expansion. The magnetic field decreases due to the expansion and we consider a general scaling $B \propto(1 / R)^{m} \propto$ $(1 / t)^{m}$ with $1 \leq m \leq 2$. When the above inequality holds, we can solve the electron kinetic equation accounting for adiabatic losses and power-law injection (with index $p_{1}$ ) of relativistic particles at a constant rate. The solution then shows the following dependencies [14, [1]]

$$
N(\gamma, R) \propto \frac{Q_{0}}{u} R\left\{\begin{array}{l}
\gamma^{-p_{1}}, \quad \gamma_{0, \text { min }}<\gamma<\gamma_{0, \text { max }} \\
\gamma^{0}, \quad \gamma_{\mathrm{R}, \text { min }}<\gamma<\gamma_{0, \text { min }},
\end{array}\right.
$$



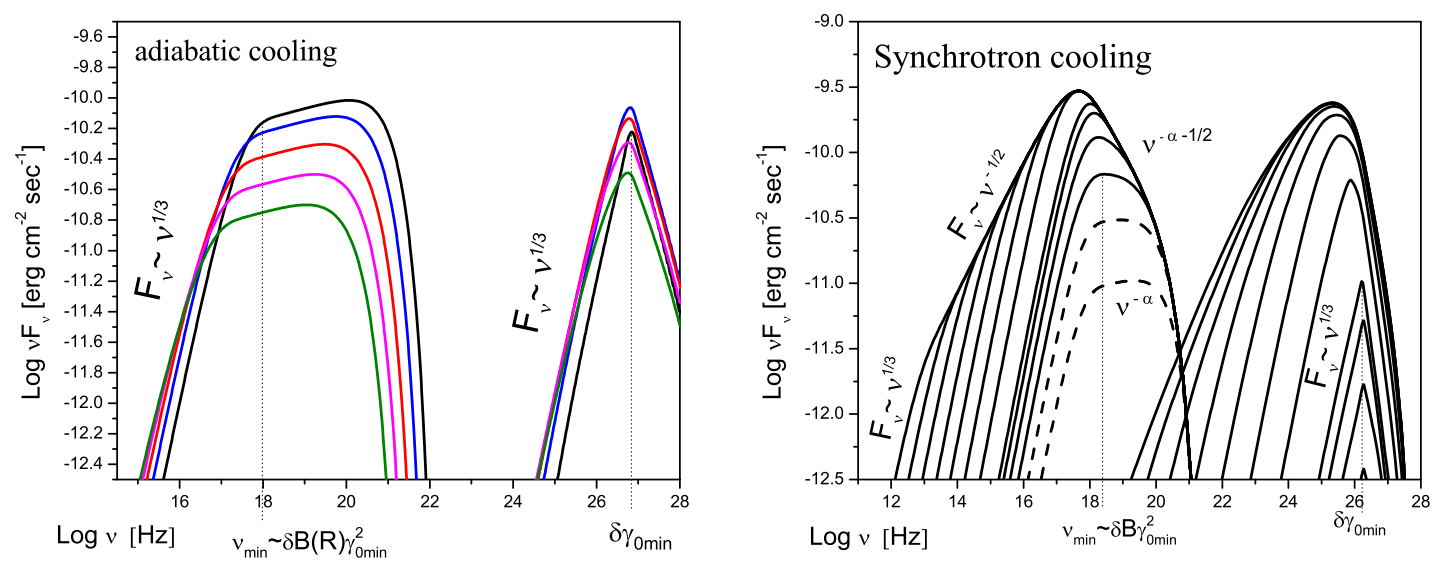

Figure 2: Evolution of the observed SSC spectrum for constant injection of a narrow power-law electron distribution, with modifications due to adiabatic cooling (left panel) and synchrotron cooling (right panel). The magnetic field is $B=0.075 \mathrm{G}$ and $B=1 \mathrm{G}$ respectively, and for the expanding source scenario we have considered a scaling $B=B_{0}\left(R_{0} / R\right)$. The initial radius is $R_{0}=7.5 \times 10^{14} \mathrm{~cm}$, expanding up to $R=10 R_{0}$ (at $u=0.1 \mathrm{c}$ ). Note that (observed) timescales for evolution are comparable for the two cases ( $t \sim 1$ day). In the adiabatic case, the hard (1/3) Compton spectral wings remains observable for longer timescales and with smaller variations in the flux. The spectrum reaches its higher flux level very quickly and then drops to lower levels until it becomes non-observable. On the contrary, for the synchrotron losses case, the luminosity increases until the source comes to a steady-state.

where $\gamma_{\mathrm{R}, \min }=\gamma_{0, \min } \frac{R_{0}}{R(t)} \propto t^{-1}$. The differential electron number density drops with radius as $n_{e}(\gamma, R)=\frac{N_{e}(\gamma, R)}{\text { Volume }} \propto R^{-2}$, and above the initial low-energy cut-off $\gamma_{0, \text { min }}$ adiabatic losses (approximately) do not modify the power-law index $n_{e}(\gamma, t) \propto \gamma^{-p_{1}}$, cf. [[20]. Below $\gamma_{0, \text { min }}$, the resulting distribution is constant with respect to the electron energies, $n_{e}(\gamma, R) \propto \gamma^{0}$. The constant part of the electron population generates a synchrotron spectrum of slope $F_{v} \propto v^{1 / 2}$ which is harder than $F_{v} \propto v^{1 / 3}$, and thus the contribution of the $\gamma_{0, \min }$-electrons (generating a $1 / 3$-synchrotron wing) shows up at lower energies (Fig. 『). For this reason, the classical hard spectrum picture at the $\mathrm{TeV}$ range can remain for timescales comparable to the source size. Even though electrons cool adiabatically as the source expands, the hard 1/3-synchrotron slope always appears below the synchrotron frequency related to the injected minimum Lorentz factor

$$
v_{\min }^{\mathrm{syn}} \propto \gamma_{0, \min }^{2} B(R) \propto \frac{1}{t^{m}}
$$

Note that any decrease of this break energy occurs due to a decrease of the magnetic field. This is different to the pure synchrotron cooling case, where the corresponding break energy follows the evolution of the minimum electron energy so that $v_{\min }^{\mathrm{syn}} \propto 1 / t^{2}$. A similar consideration applies to the energy regime where Compton scattering occurs. In particular, as long as the KN condition holds, the peak Compton energy $v_{\min }^{C}$ remains approximately constant (Fig. I). Thus, in an expanding source scenario the hard $\mathrm{TeV}$ slope is not destroyed by the cooling of the minimum cut-off electrons unlike in the synchrotron case. Of course, the decrease of the magnetic field and the luminosity of 
the source will affect its appearance - in principle, this scenario may allow for short-term variability of the hard component of the spectrum.

\section{The external Compton case}

An alternative hypothesis to the SSC scenario concerns the Comptonization of a radiation

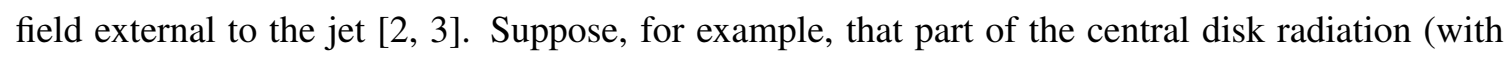
characteristic temperature $T$ ) is reprocessed/re-scattered by emission line clouds like the broad line region (BLR) and encountered by the jet. In such a case the target photon field for Compton up-scattering can appear strongly boosted in the frame of the jet.

For illustration, we show the resultant Compton spectrum for a Maxwellian electron distribution in Fig. [3. The TeV slope appears even harder than in the SSC case, with a limiting value of $F_{v} \propto v^{1}$. Any photon field which is softer (flatter) than $F_{v} \propto v^{1}$ will dominate the Compton spectrum at low energies, as in our SSC model case where the up-scattered (synchrotron) photon spectrum follows $F_{v} \propto v^{1 / 3}$. In any other case, like in the external Compton scenario with a Planckian photon field that at low energies follows $F_{v} \propto v^{2}$, the characteristic behavior of the Compton cross-section appears, implying that the radiative spectrum is dominated at its low energy part by the contribution from the up-scattering of the peak photons with $\varepsilon_{c} \sim 3 k T$.

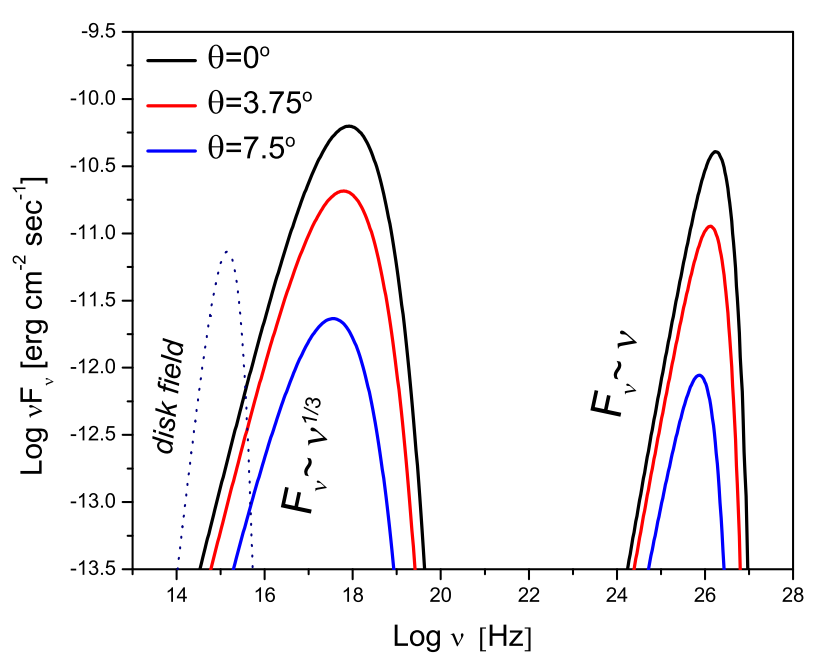

Figure 3: External Compton scenario for a quasi-Maxwellian electron distribution (with $\alpha_{p}=0$, cf. eq. [2.1.]). The observed spectrum is calculated for different angles $\theta$ to the observer. The synchrotron slope follows $F_{V} \propto v^{1 / 3}$. At the TeV range it is $F_{v} \propto v^{1}$, i.e., harder than in the SSC case. The dashed line corresponds to the assumed disk spectrum. The bulk Lorentz factor of the jet is $\Gamma=13$ and the peak energy of the electron distribution is $\gamma_{c}=2 \times 10^{4}$. For the disk photon field a temperature $T=1.75 \times 10^{4} \mathrm{~K}$ has been used. The relevant radius $R_{d}$ of the disk is considered to be of same dimensions as the jet $\left(10^{15} \mathrm{~cm}\right)$. The magnetic field is $B=1 \mathrm{G}$ and a fraction $\xi=0.1$ of the disk photons is assumed to be rescattered by the BLR. 


\section{Conclusions}

The hard, intrinsic TeV spectra of distant BL Lacs are difficult to account for with standard leptonic assumptions. The $E_{e}^{-2}$ slope that an electron energy distribution is usually thought to quickly develop due to synchrotron cooling leads to a limiting $F_{v} \propto v^{-1 / 2}$ VHE spectral shape. In addition, modifications due to Klein-Nishina effects are expected to make the TeV spectrum even steeper (softer).

As we have shown, however, if narrow, energetic electron distributions are generated within the source, standard leptonic scenarios could (partly) overcome the problems. Possible representations include Maxwellian-type distributions (as outcome of a stochastic acceleration process already balanced by cooling) or power-law distributions with high low-energy cut-offs (provided adiabatic losses dominate). The limiting value of how hard an (extended) VHE spectrum can be is $F_{v} \propto v^{1 / 3}$ for the SSC framework, and $F_{v} \propto v$ for the EC case. Note, however, that over a small energy range, achievable VHE slopes may be even harder if one allows for very high minimum cut-offs such that up-scattering occurs in the extreme $\mathrm{KN}$ regime, where the spectrum mimics the behavior of the cross-section.

\section{References}

[1] Mastichiadis, A., \& Kirk, J. G. 1997, A\&A, 320, 19

[2] Dermer, C.D., Schlickeiser, R., \& Mastichiadis, A. 1992, A\&A, 256, L27

[3] Sikora, M., Begelman, M. C., \& Rees, M. J. 1994, ApJ, 421, 153

[4] Böttcher, M., Mause, H., \& Schlickeiser, R. 1997, A\&A, 324, 395

[5] Gould, R. J., \& Schreder, G. P. 1967, Physical Review, 155, 1408

[6] Aharonian, F., et al., 2007, A\&A, 470, 475

[7] Aharonian, F., et al., 2006, Nature, 440, 1081

[8] Aharonian, F., et al., 2007, A\&A, 475, L9

[9] Aharonian, F. A., Khangulyan, D., \& Costamante, L. 2008, MNRAS, 387, 1206

[10] Böttcher, M., Dermer, C. D., \& Finke, J. D. 2008, ApJ, 679, L9

[11] Stecker, F. W., Baring, M. G. \& Summerlin, E. J. 2007, ApJL, 667, L29

[12] Aharonian, F., Timokhin, A., \& Plyasheshnikov, A. V. 2002, A\&A, 384, 834

[13] Katarzyński, K. et al. 2006, MNRAS, 368, L52

[14] Lefa, E., Rieger, F., \& Aharonian, F. 2011, to be submitted

[15] Tavecchio, F. et al. 2009, MNRAS, 399, L59

[16] Schlickeiser, R. 1985, A\&A, 143, 431

[17] Aharonian, F. A., Atoyan, A. M., \& Nahapetian, A. 1986, A\&A, 162, L1

[18] Henri, G., \& Pelletier, G. 1991, ApJL, 383, L7

[19] Atoyan A. M., \& Aharonian F. A. 1999, MNRAS, 302, 253

[20] Kardashev, N. S. 1962, Soviet Astronomy, 6, 317 